\title{
EIGENVALUES, SINGULAR VALUES, AND LITTLEWOOD-RICHARDSON COEFFICIENTS
}

\author{
SERGEY FOMIN, WILLIAM FULTON, CHI-KWONG LI, AND YIU-TUNG POON
}

\begin{abstract}
We characterize the relationship between the singular values of a Hermitian (resp., real symmetric, complex symmetric) matrix and the singular values of its offdiagonal block. We also characterize the eigenvalues of a Hermitian (or real symmetric) matrix $C=A+B$ in terms of the combined list of eigenvalues of $A$ and $B$. The answers are given by Horn-type linear inequalities. The proofs depend on a new inequality among Littlewood-Richardson coefficients.
\end{abstract}

\section{INTRODUCTION}

Let $X$ be the upper right $p$ by $n-p$ submatrix of an $n$ by $n$ matrix $Z$, with $2 p \leq n$. If $Z$ is (complex) Hermitian or real symmetric, the main result of [13] characterizes the possible eigenvalues of $Z$ in terms of the singular values of $X$. In this paper, we provide the analogous characterization for the singular values of $Z$, when $Z$ is Hermitian, or complex symmetric, or real symmetric. Surprisingly, the possibilities in all three cases are the same.

The answers are given by linear inequalities of Horn type. Let $\gamma_{1} \geq \cdots \geq \gamma_{n}$ and $s_{1} \geq \cdots \geq s_{p}$ be the singular values of $Z$ and $X$, respectively. We prove that the possible pairs of sequences $\left(s_{k}\right)$ and $\left(\gamma_{\ell}\right)$ are characterized by the inequalities

$$
2 \sum_{k \in K} s_{k} \leq \sum_{i \in I} \gamma_{2 i-1}+\sum_{j \in J} \gamma_{2 j},
$$

for all triples $(I, J, K) \in \bigcup_{r \leq p} \operatorname{LR}_{r}^{p}$, where $\operatorname{LR}_{r}^{p}$ denotes the list of triples defined inductively by Horn [9]. It appears in relation to a number of problems surveyed in [7] (where $\mathrm{LR}_{r}^{p}$ is denoted by $T_{r}^{p}$ ), including the original Horn's problem of characterizing the eigenvalues of a sum $A+B$ of two Hermitian (or real symmetric) matrices in terms of the eigenvalues of $A$ and $B$. The definition of $\mathrm{LR}_{r}^{p}$ used in this paper describes the triples $(I, J, K) \in \mathrm{LR}_{r}^{p}$ as those for which a certain Littlewood-Richardson coefficient does not vanish. See Definition 1.1.

In Section 2 of this paper, we solve the following modification of Horn's problem: what are the possible eigenvalues of $A+B$ given the combined list of eigenvalues of $A$ and $B$ (without specifying which eigenvalues belong to $A$, and which ones to $B$ )?

Our proofs depend on Klyachko's celebrated solution of Horn's problem, on its refinement obtained by Knutson and Tao, on the results on eigenvalues and singular values from [7, 8, 11, 13, and on a version of the Littlewood-Richardson rule given in [3].

Date: August 9, 2003.

1991 Mathematics Subject Classification. Primary 15A42. Secondary 05E15, 14M15, 15A18.

Partially supported by NSF grants DMS-0070685, DMS-9970435, and DMS-0071994. 


\section{Singular value inequalities}

Definition 1.1. For positive integers $r \leq p$, the set $\operatorname{LR}_{r}^{p}$ consists of all triples $(I, J, K)$ of subsets of $\{1, \ldots, p\}$ of the same cardinality $r$, such that the Littlewood-Richardson coefficient $c_{\lambda(I) \lambda(J)}^{\lambda(K)}$ is positive. (For general background on Littlewood-Richardson coefficients, see for example [6] or [4.) Here and in what follows, we use the correspondence

$$
I=\left\{i_{1}<i_{2}<\cdots<i_{r}\right\} \mapsto \lambda(I)=\left(i_{r}-r, \ldots, i_{2}-2, i_{1}-1\right)
$$

between $r$-element subsets $I$ (always written with this notation in increasing order) and integer partitions $\lambda(I)$ with at most $r$ parts.

For any complex $\ell$ by $m$ matrix $X$, there are unitary matrices $U$ and $V$ ( $\ell$ by $\ell$ and $m$ by $m$ respectively) such that $U X V$ has nonnegative entries $s_{1} \geq s_{2} \geq \ldots \geq s_{p}$ in positions $(1,1),(2,2), \ldots,(p, p)$, where $p=\min (\ell, m)$, and all other entries zero. The real numbers $s_{1}, \ldots, s_{p}$, always written in decreasing order, with multiplicities, are the singular values of $X$. The positive singular values are the positive square roots of the eigenvalues of the positive semidefinite matrix $X^{*} X$, where $X^{*}$ denotes the conjugate transpose of $X$. If $X$ is Hermitian, then its singular values are simply the absolute values of its eigenvalues.

1.1. Main result. The following is our main result on singular values.

Theorem 1.2. Let $p$ and $n$ be positive integers, with $2 p \leq n$, and let $\gamma_{1} \geq \cdots \geq \gamma_{n}$ and $s_{1} \geq \cdots \geq s_{p}$ be sequences of nonnegative real numbers. The following are equivalent:

(a) There exists a complex matrix $Z$ of the form $Z=\left[\begin{array}{c}* \\ Y \\ x_{*}^{*}\end{array}\right]$ such that

- $X$ is $p$ by $n-p$, with singular values $s_{1}, \ldots, s_{p}$;

- $Y$ is $n-p$ by $p$, with singular values $s_{1}, \ldots, s_{p}$;

- $Z$ is $n$ by $n$, with singular values $\gamma_{1}, \ldots, \gamma_{n}$.

(b) There exists a real symmetric matrix $Z=\left[\begin{array}{c}* \\ X^{*}\end{array}{ }_{*}^{*}\right]$ such that

- $X$ is $p$ by $n-p$, with singular values $s_{1}, \ldots, s_{p}$;

- $Z$ is $n$ by $n$, with singular values $\gamma_{1}, \ldots, \gamma_{n}$ (thus, eigenvalues $\pm \gamma_{1}, \ldots, \pm \gamma_{n}$ ).

(c) There exists a real symmetric matrix $Z=\left[\begin{array}{cc}* & X \\ X^{*} & *\end{array}\right]$ such that

- $X$ is $p$ by $n-p$, with singular values $s_{1}, \ldots, s_{p}$;

- $Z$ is $n$ by $n$, with eigenvalues $\gamma_{1},-\gamma_{2}, \gamma_{3},-\gamma_{4}, \ldots,(-1)^{n-1} \gamma_{n}$.

(d) The numbers $\gamma_{1}, \ldots, \gamma_{2 p}$ and $s_{1}, \ldots, s_{p}$ satisfy the linear inequalities

$$
2 \sum_{k \in K} s_{k} \leq \sum_{i \in I} \gamma_{2 i-1}+\sum_{j \in J} \gamma_{2 j}
$$

for all $r \leq p$ and all triples $(I, J, K) \in \mathrm{LR}_{r}^{p}$.

The equivalence of (ㄷ) and (d) is a direct corollary of the main results in [8] and [13] (see Section 1.2 below). The other equivalences are new. Conditions (ad) -(d) are also equivalent to condition (国) in Corollary 1.8, and to condition (If) in Corollary 2.4. 
Remark 1.3. In (国), "complex" can be replaced by "complex symmetric" or "Hermitian." Indeed, the resulting statements imply (国) and are implied by (b). In these versions, the somewhat unnatural requirement that $X$ and $Y$ have the same singular values becomes redundant. See also Remark 1.20.

Remark 1.4. Condition (d d ) does not involve the $\gamma_{i}$ with $i>2 p$. Hence in (国)-(值), one can replace " $Z$ is $n$ by $n$, with singular values $\gamma_{1}, \ldots, \gamma_{n}$ " by " $Z$ is $n$ by $n$, with largest $2 p$ singular values $\gamma_{1}, \ldots, \gamma_{2 p} . "$

Remark 1.5. In (d) , it suffices to check the inequalities for the triples $(I, J, K)$ with $c_{\lambda(I) \lambda(J)}^{\lambda(K)}=1$. This follows from a theorem of P. Belkale (see [7, Proposition 9]).

Remark 1.6. The matrices $X$ and $Y$ in (国) can be specified in advance, as can the matrix $X$ in (b) -( (c) ). Indeed, any two matrices with the same singular values can be transformed into each other by multiplying on the left and right by unitary (orthogonal in the real case) matrices. On the other hand, for unitary matrices $U_{1}, U_{2}, V_{1}$, and $V_{2}$, the matrix

$$
\left[\begin{array}{cc}
U_{1} & 0 \\
0 & U_{2}
\end{array}\right] \cdot\left[\begin{array}{cc}
P & X \\
Y & Q
\end{array}\right] \cdot\left[\begin{array}{cc}
V_{1} & 0 \\
0 & V_{2}
\end{array}\right]=\left[\begin{array}{ll}
U_{1} P V_{1} & U_{1} X V_{2} \\
U_{2} Y V_{1} & U_{2} Q V_{2}
\end{array}\right]
$$

has the same singular values as $Z=\left[\begin{array}{cc}P & X \\ Y & Q\end{array}\right]$.

1.2. Comparison with previous results. Examples. We next summarize the main result of [13], which includes the main result of [8], in a form suitable for our purposes.

Theorem 1.7. Let $2 p \leq n$, let $s_{1} \geq \ldots \geq s_{p} \geq 0$, and $\lambda_{1} \geq \ldots \geq \lambda_{n}$. The following are equivalent:

(i) There exists an $n$ by $n$ Hermitian matrix of the form $\left[\begin{array}{c}* \\ X^{*} \\ *\end{array}\right]$ with eigenvalues $\lambda_{1}, \ldots, \lambda_{n}$, such that $X$ is $p$ by $n-p$ with singular values $s_{1}, \ldots, s_{p}$.

(ii) For all $r \leq p$ and $(I, J, K)$ in $\mathrm{LR}_{r}^{p}$,

$$
2 \sum_{k \in K} s_{k} \leq \sum_{i \in I} \lambda_{i}-\sum_{j \in J} \lambda_{n+1-j} .
$$

(iii) There exist Hermitian $p$ by $p$ matrices $A, B$, and $C$ with eigenvalues $\lambda_{1}, \ldots, \lambda_{p}$, $\lambda_{n+1-p}, \ldots, \lambda_{n}$, and $s_{1}, \ldots, s_{p}$, respectively, such that $2 C \leq A-B$, i.e., $A-B-2 C$ is positive semidefinite.

(iv) There exist Hermitian $p$ by $p$ matrices $A, B$, and $C$ such that $2 C \leq A-B$, the eigenvalues of $C$ are $s_{1}, \ldots, s_{p}$, and the eigenvalues of $A$ (respectively, $B$ ) listed in descending order (with multiplicities) form a subsequence of $\lambda_{1}, \ldots, \lambda_{n}$.

The Hermitian matrices in (i), (iii), and (iv) can be taken to be real symmetric matrices, and the matrices $X$ in (i) and $C$ in (iii) and (iv) can be specified in advance.

The equivalences (i)-(iii) in Theorem [1.7 are essentially [13, Theorem 2.3]. The proof of Theorem 1.7 is given in Section 3 . 
Corollary 1.8. In Theorem [1.2, conditions (드) and (d $)$ are equivalent to each other, and also to

(e) There exist Hermitian $p$ by $p$ matrices $A, B$, and $C$ with eigenvalues $\gamma_{1}, \gamma_{3}, \ldots, \gamma_{2 p-1}$ (of $A$ ), $\gamma_{2}, \gamma_{4}, \ldots, \gamma_{2 p}$ (of $B$ ), and $s_{1}, s_{2}, \ldots, s_{p}$ (of $C$ ), such that $2 C \leq A+B$.

Proof. Apply the equivalence (i) $\Leftrightarrow$ (iii) of Theorem [1.7 with $\left(\lambda_{1}, \lambda_{2}, \ldots, \lambda_{n-1}, \lambda_{n}\right)=$ $\left(\gamma_{1}, \gamma_{3}, \ldots,-\gamma_{4},-\gamma_{2}\right)$. (Here in the right-hand side, the numbers $(-1)^{i-1} \gamma_{i}$ are listed in decreasing order.)

Remark 1.9. Condition (国) can be further relaxed - see condition (If) in Corollary 2.4

Remark 1.10. Condition (b) of Theorem 1.2 concerns real symmetric (or Hermitian) matrices with eigenvalues $\pm \gamma_{1}, \ldots, \pm \gamma_{n}$. There are $2^{n}$ choices of signs, and for each choice of signs, Theorem [1.7 describes the relationship between the possible values of $\gamma_{1}, \ldots, \gamma_{n}$ and $s_{1}, \ldots, s_{p}$ by a set of linear inequalities (ii). The assertion (b) $\Leftrightarrow$ (IC) in Theorem 1.2 can be rephrased as saying that the set of inequalities corresponding to the alternating choice of signs $\left(\gamma_{1},-\gamma_{2}, \gamma_{3},-\gamma_{4}, \ldots\right)$ is uniformly the weakest. That is, the inequalities (ii) corresponding to any other choice of signs imply the inequalities (2).

Remark 1.11. Removing the restrictions concerning matrix $Y$ in part (国) of Theorem 1.2 results in a different (strictly weaker than (d) ) - and much simpler - set of inequalities. Specifically, it is not hard to prove (cf. a more general result of Thompson's 17]) that, for $2 p \leq n$, there exists a $p$ by $n-p$ matrix with singular values $s_{1} \geq \cdots \geq s_{p}$ that is a submatrix of an $n$ by $n$ matrix with singular values $\gamma_{1} \geq \cdots \geq \gamma_{n}$ if and only if $\gamma_{i} \geq s_{i}$ for $i=1, \ldots, p$.

Remark 1.12. O'Shea and Sjamaar [15] give another polyhedral description of the singular values appearing in part (B) of Theorem 1.2. However, 15] contains no inequalities like (2). We do not know whether the equivalence $(\mathrm{b}) \Leftrightarrow(\underline{\mathrm{d}})$ can be deduced from [15].

Example 1.13. Let $p=1$. The only triple $(I, J, K) \in \operatorname{LR}_{1}^{1}$ is $(\{1\},\{1\},\{1\})$. The corresponding inequality (2) is

$$
2 s_{1} \leq \gamma_{1}+\gamma_{2}
$$

In the special case $p=1, n=2$, the equivalence $($ a $) \Leftrightarrow($ did $)$ in Theorem 1.2 can be restated as follows: the singular values $\gamma_{1} \geq \gamma_{2} \geq 0$ of a 2 by 2 complex matrix of the form $\left[\begin{array}{ll}* & x \\ y & *\end{array}\right]$, where $|x|=|y|=s_{1} \geq 0$ is fixed, satisfy the inequality (44) and no other constraints. Equivalently (see Remark 1.6), the inequality (4) describes the possible singular values $\gamma_{1}, \gamma_{2}$ of a 2 by 2 complex (or real) symmetric matrix $Z$ of the form $Z=\left[\begin{array}{cc}* & s_{1} \\ s_{1} & *\end{array}\right]$. To compare, Theorem [1.7 asserts that the eigenvalues $\lambda_{1} \geq \lambda_{2}$ of $Z$ satisfy $2 s_{1} \leq \lambda_{1}-\lambda_{2}$ (and, generally speaking, nothing else), which translates into the restrictions (4) for the singular values $\gamma_{1}=\left|\lambda_{1}\right|$ and $\gamma_{2}=\left|\lambda_{2}\right|$. 
Example 1.14. For $p=2$, the triples $(I, J, K)$ and the corresponding inequalities (2) are:

$$
\begin{aligned}
(\{1\},\{1\},\{1\}) & 2 s_{1} & \leq \gamma_{1}+\gamma_{2} \\
(\{1\},\{2\},\{2\}) & 2 s_{2} & \leq \gamma_{1}+\gamma_{4} \\
(\{2\},\{1\},\{2\}) & 2 s_{2} & \leq \gamma_{2}+\gamma_{3} \\
(\{1,2\},\{1,2\},\{1,2\}) & 2\left(s_{1}+s_{2}\right) & \leq \gamma_{1}+\gamma_{2}+\gamma_{3}+\gamma_{4}
\end{aligned}
$$

Thus, in the special case $p=2, n=4$, the equivalence (国) $\Leftrightarrow$ (did) means that the singular values $\gamma_{1} \geq \gamma_{2} \geq \gamma_{3} \geq \gamma_{4} \geq 0$ of a 4 by 4 complex matrix of the form $Z=\left[\begin{array}{ll}* & X \\ Y & *\end{array}\right]$ whose 2 by 2 blocks $X$ and $Y$ have fixed singular values $s_{1} \geq s_{2}$ satisfy (5) and no other constraints. To compare, Theorem 1.7 provides the following list of inequalities relating the eigenvalues $\lambda_{1} \geq \lambda_{2} \geq \lambda_{3} \geq \lambda_{4}$ of a Hermitian $Z$ to the singular values $s_{1}$ and $s_{2}$ of $X$ and $Y$ :

$$
\begin{aligned}
& (\{1\},\{1\},\{1\}) \\
& (\{1\},\{2\},\{2\}) \\
& 2 s_{1} \leq \lambda_{1}-\lambda_{4} \\
& (\{2\},\{1\},\{2\}) \\
& 2 s_{2} \leq \lambda_{1}-\lambda_{3} \\
& 2 s_{2} \leq \lambda_{2}-\lambda_{4} \\
& (\{1,2\},\{1,2\},\{1,2\}) \\
& 2\left(s_{1}+s_{2}\right) \leq \lambda_{1}+\lambda_{2}-\lambda_{3}-\lambda_{4}
\end{aligned}
$$

To illustrate Remark 1.10, for fixed singular values $\gamma_{1}, \ldots, \gamma_{4}$, there are 16 choices of signs for the eigenvalues $\pm \gamma_{1}, \ldots, \pm \gamma_{4}$. For each of these choices, sorting the eigenvalues in decreasing order produces a list $\left(\lambda_{1}, \ldots, \lambda_{4}\right)$. The resulting inequalities are always (weakly) stronger than (5). (The latter corresponds to $\left(\lambda_{1}, \lambda_{2}, \lambda_{3}, \lambda_{4}\right)=\left(\gamma_{1}, \gamma_{3},-\gamma_{4},-\gamma_{2}\right)$.) For example, if the eigenvalues are $-\gamma_{1}, \gamma_{2}, \gamma_{3},-\gamma_{4}$, then $\left(\lambda_{1}, \lambda_{2}, \lambda_{3}, \lambda_{4}\right)=\left(\gamma_{2}, \gamma_{3},-\gamma_{4},-\gamma_{1}\right)$, and (6) yields four inequalities

$$
2 s_{1} \leq \gamma_{1}+\gamma_{2}, \quad 2 s_{2} \leq \gamma_{2}+\gamma_{4}, \quad 2 s_{2} \leq \gamma_{1}+\gamma_{3}, \quad 2\left(s_{1}+s_{2}\right) \leq \gamma_{1}+\gamma_{2}+\gamma_{3}+\gamma_{4},
$$

which collectively imply (15).

1.3. Singular value inequalities for arbitrary $X$ and $Y$. It is natural to ask whether the restriction in Theorem 1.2 that $X$ and $Y$ have the same singular values can be removed (with some other collection of inequalities playing the role of (2)).

Problem 1.15. Find necessary and sufficient conditions for the existence of matrices $X, Y$, and $Z=\left[\begin{array}{c}* \\ Y \\ *\end{array}\right]$ with given singular values. Can those conditions be given by a collection of linear inequalities?

In Proposition 1.16 below, we provide a set of necessary (but not sufficient) conditions for this problem. These conditions, however, will turn out to be sufficient in the special case considered in Theorem [1.2, and will play a role in the proof of the latter.

Recall that the triples of sequences of nonnegative real numbers $\left(a_{1} \geq \cdots \geq a_{n}\right)$, $\left(b_{1} \geq \cdots \geq b_{n}\right),\left(c_{1} \geq \cdots \geq c_{n}\right)$ that can occur as singular values of complex $n$ by $n$ 
matrices $A, B$, and $C=A+B$ are also characterized by a list of linear inequalities. More specifically, note that the $2 n$ by $2 n$ matrices appearing in the identity

$$
\left[\begin{array}{cc}
0 & A \\
A^{*} & 0
\end{array}\right]+\left[\begin{array}{cc}
0 & B \\
B^{*} & 0
\end{array}\right]=\left[\begin{array}{cc}
0 & C \\
C^{*} & 0
\end{array}\right]
$$

have eigenvalues $a_{1} \geq \cdots \geq a_{n} \geq-a_{n} \geq \cdots \geq-a_{1}, b_{1} \geq \cdots \geq b_{n} \geq-b_{n} \geq \cdots \geq-b_{1}$, $c_{1} \geq \cdots \geq c_{n} \geq-c_{n} \geq \cdots \geq-c_{1}$, respectively, and therefore these three sequences must satisfy the Horn inequalities (see Proposition 2.1). Conversely, those inequalities are sufficient for the existence of $A, B$, and $C=A+B$ with the desired singular values; see [1] for the proof, and [7, Section 5] for a detailed exposition.

Proposition 1.16. Let $2 p \leq n$, and let $Z=\left[\begin{array}{cc}P & X \\ Y & Q\end{array}\right]$ be a complex matrix such that

- $X$ is $p$ by $n-p$, with singular values $s_{1} \geq \cdots \geq s_{p}$;

- $Y$ is $n-p$ by $p$, with singular values $t_{1} \geq \cdots \geq t_{p}$;

- $Z$ is $n$ by $n$, with singular values $\gamma_{1} \geq \cdots \geq \gamma_{n}$.

Let $\sigma_{1} \geq \cdots \geq \sigma_{2 p}$ be the decreasing rearrangement of the numbers $s_{1}, \ldots, s_{p}, t_{1}, \ldots, t_{p}$. Then, for all $1 \leq m<2 n$ and all triples $(E, F, G) \in \mathrm{LR}_{m}^{2 n}$,

$$
2\left(\sum_{\substack{g \in G \\ g \leq 2 p}} \sigma_{g}-\sum_{\substack{g \in G^{\prime} \\ g \leq 2 p}} \sigma_{g}\right) \leq \sum_{\substack{e \in E \\ e \leq n}} \gamma_{e}-\sum_{\substack{e \in E^{\prime} \\ e \leq n}} \gamma_{e}+\sum_{\substack{f \in F \\ f \leq n}} \gamma_{f}-\sum_{\substack{f \in F^{\prime} \\ f \leq n}} \gamma_{f}
$$

where we denote

$$
G^{\prime}=\{g \in\{1, \ldots, 2 n\} \mid 2 n+1-g \in G\},
$$

and similarly for $E$ and $F$.

In particular, for any triple of the form $(F, F, G) \in \mathrm{LR}_{m}^{2 n}$, with $m<2 n$,

$$
\sum_{\substack{g \in G \\ g \leq 2 p}} \sigma_{g}-\sum_{\substack{g \in G^{\prime} \\ g \leq 2 p}} \sigma_{g} \leq \sum_{\substack{f \in F \\ f \leq n}} \gamma_{f}-\sum_{\substack{f \in F^{\prime} \\ f \leq n}} \gamma_{f}
$$

Proof. In the identity

$$
\left[\begin{array}{cc}
P & X \\
Y & Q
\end{array}\right]+\left[\begin{array}{cc}
-P & X \\
Y & -Q
\end{array}\right]=2\left[\begin{array}{cc}
0 & X \\
Y & 0
\end{array}\right]
$$

both matrices on the left-hand side have singular values $\gamma_{1} \geq \ldots \geq \gamma_{n}$, as one sees by applying (3) with $U_{1}=V_{1}=\sqrt{-1} I_{p}$ and $U_{2}=V_{2}=-\sqrt{-1} I_{n-p}$. The right-hand side has ordered singular values $2 \sigma_{1}, \ldots, 2 \sigma_{2 p}, 0, \ldots, 0$. Applying (the easy part of) [7. Theorem 15] to the matrices in (9), we obtain (71).

The converse of Proposition 1.16 is false. That is, the inequalities (17) are necessary but not sufficient for the existence of a matrix $Z$ with described properties. See Example 1.17. 
Example 1.17. Let $p=1$ and $n=2$. Thus, we fix $s_{1} \geq 0, t_{1} \geq 0$, and $\gamma_{1} \geq \gamma_{2} \geq 0$, and look at the matrices $Z=\left[\begin{array}{cc}p & x \\ y & q\end{array}\right]$ with $|x|=s_{1},|y|=t_{1}$, and with singular values $\gamma_{1}$ and $\gamma_{2}$. By the definition of singular values, such a matrix exists if and only if there are complex numbers $p$ and $q$ such that

$$
\begin{aligned}
\gamma_{1}^{2}+\gamma_{2}^{2} & =|p|^{2}+|q|^{2}+|x|^{2}+|y|^{2}, \\
\gamma_{1} \gamma_{2} & =|p q-x y|
\end{aligned}
$$

for some $x$ and $y$ with $|x|=s_{1}$ and $|y|=t_{1}$. Equivalently,

$$
\begin{gathered}
\quad \exists p, q \in \mathbb{C}\left\{\begin{array}{c}
\gamma_{1}^{2}+\gamma_{2}^{2}=|p|^{2}+|q|^{2}+s_{1}^{2}+t_{1}^{2} \\
\gamma_{1} \gamma_{2}=\left|p q-s_{1} t_{1}\right|
\end{array}\right. \\
\Longleftrightarrow \quad \exists p, q \in \mathbb{C}\left\{\begin{array}{c}
|p|^{2}+|q|^{2}=\gamma_{1}^{2}+\gamma_{2}^{2}-s_{1}^{2}-t_{1}^{2} \\
\left|\gamma_{1} \gamma_{2}-s_{1} t_{1}\right| \leq|p q| \leq\left|\gamma_{1} \gamma_{2}+s_{1} t_{1}\right|
\end{array}\right. \\
\Longleftrightarrow \quad 2\left|\gamma_{1} \gamma_{2}-s_{1} t_{1}\right| \leq \gamma_{1}^{2}+\gamma_{2}^{2}-s_{1}^{2}-t_{1}^{2}
\end{gathered}
$$

Thus, in this special case, the necessary and sufficient conditions for the existence of such matrix $Z$ are given by the linear inequalities (10). (This is precisely [18, Lemma 1].) On the other hand, the only essential inequalities among (71) are the ones corresponding to $E=F=G=\{1\}$ and $E=F=G=\{1,2\}$; they are, respectively, $\sigma_{1} \leq \gamma_{1}$ and $\sigma_{1}+\sigma_{2} \leq \gamma_{1}+\gamma_{2}$. Equivalently,

$$
\begin{aligned}
\max \left(s_{1}, t_{1}\right) & \leq \gamma_{1}, \\
s_{1}+t_{1} & \leq \gamma_{1}+\gamma_{2} .
\end{aligned}
$$

Since the inequalities (11) do not imply (10), the converse of Proposition 1.16 fails.

1.4. Inequalities for Littlewood-Richardson coefficients. Our proof of Theorem 1.2 (see Section [1.5) is based on Theorem [1.7. Proposition 1.16, and the following lemma, proved combinatorially in Section 4 using a result of Carré and Leclerc [3].

Lemma 1.18. If $(I, J, K) \in \mathrm{LR}_{r}^{p}$, then $(F, F, G) \in \mathrm{LR}_{2 r}^{2 p}$, where

$$
\begin{aligned}
& F=\left\{2 i_{1}-1, \ldots, 2 i_{r}-1\right\} \cup\left\{2 j_{1}, \ldots, 2 j_{r}\right\}, \\
& G=\left\{2 k_{1}-1, \ldots, 2 k_{r}-1\right\} \cup\left\{2 k_{1}, \ldots, 2 k_{r}\right\} .
\end{aligned}
$$


If $\lambda$ and $\mu$ are the partitions associated to $I$ and $J$ by the correspondence (11), let $\tau(\lambda, \mu)$ be the partition corresponding to the set $F$ defined by (12). The lemma says that if $c_{\lambda, \mu}^{\nu}$ is positive, then $c_{\tau(\lambda, \mu), \tau(\lambda, \mu)}^{\tau(\nu, \nu)}$ is also positive. In Section 4 we prove the following stronger assertion (see Proposition 4.5):

Proposition 1.19. If $\lambda, \mu$, and $\nu$ satisfy $|\lambda|+|\mu|=|\nu|$, then $c_{\lambda, \mu}^{\nu} \leq c_{\tau(\lambda, \mu), \tau(\lambda, \mu)}^{\tau(\nu, \nu)}$.

The correspondence $(\lambda, \mu) \mapsto \tau(\lambda, \mu)$ is a disguised version of (the inverse of) the 2quotient map, well known in algebraic combinatorics of Young diagrams. See more about this in Section 4. A stronger but unproved inequality between Littlewood-Richardson coefficients (see Conjecture 5.1) is discussed in Section 5.

1.5. Proof of Theorem 1.2 modulo Theorem 1.7 and Proposition 1.19. The implications $(\mathbb{C}) \Rightarrow(\mathbb{b}) \Rightarrow($ a $)$ in Theorem 1.2 are trivial. The equivalence $(\mathbb{C}) \Leftrightarrow(\mathbb{d})$ was proved in Section 1.2 using Theorem 1.7. It remains to prove that (国) $\Rightarrow$ (d) .

Let $(I, J, K) \in \mathrm{LR}_{r}^{p}$, and let $F$ and $G$ be given by (12)-(13). By Lemma 1.18 (which follows from Proposition 1.19), $(F, F, G) \in \mathrm{LR}_{2 r}^{2 p} \subset \mathrm{LR}_{2 r}^{n}$. We next apply Proposition 1.16. with $t_{k}=s_{k}$ and $\sigma_{2 k-1}=\sigma_{2 k}=s_{k}$ for $k=1, \ldots, p$. Observing that the negative sums on both sides of (8) disappear for $F, G \subset\{1, \ldots, n\}$, we obtain:

$$
\sum_{k \in K} s_{k}+\sum_{k \in K} s_{k}=\sum_{g \in G} \sigma_{g} \leq \sum_{f \in F} \gamma_{f}=\sum_{i \in I} \gamma_{2 i-1}+\sum_{j \in J} \gamma_{2 j}
$$

as desired.

Remark 1.20. The argument in the proof above can be adapted to show that condition (ai) of Theorem 1.2 can be replaced by

(미) There exists an $n$ by $n$ complex matrix $\left[\begin{array}{c}* \\ Y\end{array}\right]$ with singular values $\gamma_{1}, \ldots, \gamma_{n}$ such that the matrix $\left[\begin{array}{cc}0 & X \\ Y & 0\end{array}\right]$ has singular values $s_{1}, s_{1}, s_{2}, s_{2}, \ldots, s_{p}, s_{p}$.

(Here, as before, $X$ and $Y$ are $p$ by $n-p$ and $n-p$ by $p$, respectively.)

1.6. Outline of the rest of the paper. In Section 2, we use Lemma 1.18 to characterize the possible eigenvalues of a matrix obtained as a sum of two Hermitian matrices with a given combined list of eigenvalues. In turn, this result leads to (apparently) new statements concerning Littlewood-Richardson coefficients.

Sections 3 and 4 contain the proofs of Theorem 1.7 and Proposition 1.19, respectively.

Although not required for our proof, we include in Section 5] a geometric argument that deduces Proposition 1.19 from a stronger inequality (see Conjecture 5.10) for the Littlewood-Richardson coefficients. For each ordered pair $(\lambda, \mu)$ of partitions, a simple rule produces another pair $\left(\lambda^{*}, \mu^{*}\right)$, with $\left|\lambda^{*}\right|+\left|\mu^{*}\right|=|\lambda|+|\mu|$. We conjecture that $c_{\lambda^{*} \mu^{*}}^{\nu} \geq c_{\lambda \mu}^{\nu}$ for all partitions $\nu$. While some cases of this conjecture can be deduced from known matrix identities, the general case seems to require new ideas. 


\section{HoRn'S PROBLEM FOR A COMBINED LIST OF EIGENVALUES}

Throughout this section, "Hermitian" can be replaced by "real symmetric."

For any $p$-element lists $a=\left(a_{1} \geq \cdots \geq a_{p}\right)$ and $b=\left(b_{1} \geq \cdots \geq b_{p}\right)$ of real numbers, let $\mathbf{H}(a ; b)$ denote the set of $p$ by $p$ matrices $C$ that can be expressed as $C=A+B$, where $A$ and $B$ are Hermitian matrices with eigenvalues $a$ and $b$, respectively. By the celebrated conjecture of A. Horn's (proved by A. A. Klyachko and A. Knutson-T. Tao, see [7. Section 1]), the set $\mathbf{H}(a ; b)$ is described as follows.

Proposition 2.1. $\mathbf{H}(a ; b)$ consists of the matrices $C$ whose eigenvalues $c_{1} \geq \cdots \geq c_{p}$ satisfy the trace condition $\sum_{i} c_{i}=\sum_{i} a_{i}+\sum_{i} b_{i}$, together with the Horn inequalities

$$
\sum_{k \in K} c_{k} \leq \sum_{i \in I} a_{i}+\sum_{j \in J} b_{j}
$$

for all $r<p$ and all triples $(I, J, K)$ in $\operatorname{LR}_{r}^{p}$.

Now suppose that rather than fixing the lists $a$ and $b$, we only fix their union $\gamma=$ $\left(\gamma_{1} \geq \cdots \geq \gamma_{2 p}\right)=a \sqcup b$ (taken with multiplicities). Which matrices $C$ can be written as a sum of two matrices whose joint list of eigenvalues is $\gamma$ ? According to Proposition 2.2 below, the answer is given by the set

$$
\mathbf{H}\left(\gamma_{1}, \gamma_{3}, \ldots, \gamma_{2 p-1} ; \gamma_{2}, \gamma_{4}, \ldots, \gamma_{2 p}\right) .
$$

In other words, any other splitting of $\gamma$ into two $p$-element sublists $a$ and $b$ produces a set $\mathbf{H}(a ; b)$ that is contained in (15).

Proposition 2.2. Let $A$ and $B$ be $p$ by $p$ Hermitian matrices. Let $\gamma_{1} \geq \cdots \geq \gamma_{2 p}$ be the eigenvalues of $A$ and $B$ arranged in descending order. Then there exist Hermitian matrices $\widetilde{A}$ and $\widetilde{B}$ with eigenvalues $\gamma_{1}, \gamma_{3}, \ldots, \gamma_{2 p-1}$ and $\gamma_{2}, \gamma_{4}, \ldots, \gamma_{2 p}$, respectively, such that $\widetilde{A}+\widetilde{B}=A+B$.

Although Proposition 2.2 can be deduced by comparing the equivalence $(\mathrm{b}) \Leftrightarrow(\mathbf{C})$ of Theorem [1.2 with Theorem [1.7 we provide a shortcut proof below, based directly on Lemma 1.18

Proof. Let $r \leq p$, and suppose that $(I, J, K) \in \mathrm{LR}_{r}^{p}$. Let $F$ and $G$ be given by (12). By Lemma 1.18, $(F, F, G) \in \mathrm{LR}_{2 r}^{2 p}$. Applying the corresponding Horn inequality to the identity

we obtain

$$
\left[\begin{array}{cc}
A & 0 \\
0 & B
\end{array}\right]+\left[\begin{array}{cc}
B & 0 \\
0 & A
\end{array}\right]=\left[\begin{array}{cc}
A+B & 0 \\
0 & A+B
\end{array}\right]
$$

$$
2\left(\sum_{i \in I} \gamma_{2 i-1}+\sum_{j \in J} \gamma_{2 j}\right) \geq 2 \sum_{k \in K} c_{k},
$$

where $c_{1}, \ldots, c_{p}$ are the eigenvalues of $C=A+B$. Since $\sum \gamma_{2 i-1}+\sum \gamma_{2 j}=\sum c_{i}$ as well, the claim follows by Proposition 2.1. 
Example 2.3. Let $p=2$. We are looking at matrices $C$ that can be expressed as $A+B$, where $A$ and $B$ have the joint list of eigenvalues $\gamma=\left(\gamma_{1} \geq \gamma_{2} \geq \gamma_{3} \geq \gamma_{4}\right)$. The eigenvalues $c_{1} \geq c_{2}$ of $C$ must satisfy $c_{1}+c_{2}=\sum \gamma_{i}$, along with the inequalities (14), which depending on the splitting of $\gamma$ into $a=\left(a_{1} \geq a_{2}\right)$ and $b=\left(b_{1} \geq b_{2}\right)$, will take the following form:

\begin{tabular}{clll}
$I, J, K$ & $a=\left(\gamma_{1}, \gamma_{3}\right)$ & $a=\left(\gamma_{1}, \gamma_{4}\right)$ & $a=\left(\gamma_{1}, \gamma_{2}\right)$ \\
& $b=\left(\gamma_{2}, \gamma_{4}\right)$ & $b=\left(\gamma_{2}, \gamma_{3}\right)$ & $b=\left(\gamma_{3}, \gamma_{4}\right)$ \\
\hline$\{1\},\{1\},\{1\}$ & $c_{1} \leq \gamma_{1}+\gamma_{2}$ & $c_{1} \leq \gamma_{1}+\gamma_{2}$ & $c_{1} \leq \gamma_{1}+\gamma_{3}$ \\
$\{1\},\{2\},\{2\}$ & $c_{2} \leq \gamma_{1}+\gamma_{4}$ & $c_{2} \leq \gamma_{1}+\gamma_{3}$ & $c_{2} \leq \gamma_{1}+\gamma_{4}$ \\
$\{2\},\{1\},\{2\}$ & $c_{2} \leq \gamma_{2}+\gamma_{3}$ & $c_{2} \leq \gamma_{2}+\gamma_{4}$ & $c_{2} \leq \gamma_{2}+\gamma_{3}$
\end{tabular}

Replacing $c_{2}$ by $\gamma_{1}+\cdots+\gamma_{4}-c_{1}$, we obtain the following conditions for $c_{1}$, for each of the three possible splittings shown in (16):

\begin{tabular}{ccc}
$a=\left(\gamma_{1}, \gamma_{3}\right)$ & $a=\left(\gamma_{1}, \gamma_{4}\right)$ & $a=\left(\gamma_{1}, \gamma_{2}\right)$ \\
$b=\left(\gamma_{2}, \gamma_{4}\right)$ & $b=\left(\gamma_{2}, \gamma_{3}\right)$ & $b=\left(\gamma_{3}, \gamma_{4}\right)$ \\
\hline$c_{1} \leq \gamma_{1}+\gamma_{2}$ & $c_{1} \leq \gamma_{1}+\gamma_{2}$ & $c_{1} \leq \gamma_{1}+\gamma_{3}$ \\
$c_{1} \geq \max \left(\gamma_{2}+\gamma_{3}, \gamma_{1}+\gamma_{4}\right)$ & $c_{1} \geq \gamma_{1}+\gamma_{3}$ & $c_{1} \geq \max \left(\gamma_{2}+\gamma_{3}, \gamma_{1}+\gamma_{4}\right)$
\end{tabular}

It is easy to see that the conditions in the first column are the least restrictive among the three sets. To give a concrete example, take $\gamma_{1}=4, \gamma_{2}=3, \gamma_{3}=2, \gamma_{4}=1$. Then

$$
\begin{aligned}
& \mathbf{H}(4,2 ; 3,1)=\left\{C \mid c_{1} \in[5,7], c_{1}+c_{2}=10\right\}, \\
& \mathbf{H}(4,1 ; 3,2)=\left\{C \mid c_{1} \in[6,7], c_{1}+c_{2}=10\right\}, \\
& \mathbf{H}(4,3 ; 2,1)=\left\{C \mid c_{1} \in[5,6], c_{1}+c_{2}=10\right\} .
\end{aligned}
$$

Proposition 2.2 has the following direct implication.

Corollary 2.4. Condition (國) of Corollary 1.8 is equivalent to

(f) There exist (positive semidefinite) Hermitian $p$ by $p$ matrices $A, B$, and $C$ such that: $\left[\begin{array}{ll}A & 0 \\ 0 & B\end{array}\right]$ has eigenvalues $\gamma_{1}, \ldots, \gamma_{2 p} ; C$ has eigenvalues $s_{1}, \ldots, s_{p} ;$ and $2 C \leq$ $A+B$.

Corollary 2.5. For a pair $(\lambda, \mu)$ of partitions, let $\gamma_{1} \geq \cdots \geq \gamma_{2 p}$ be the decreasing rearrangement of the $\lambda_{i}$ and $\mu_{j}$ 's. Define two partitions

$$
\widetilde{\lambda}=\left(\gamma_{1}, \gamma_{3}, \ldots, \gamma_{2 p-1}\right), \quad \widetilde{\mu}=\left(\gamma_{2}, \gamma_{4}, \ldots, \gamma_{2 p}\right) .
$$

Then for every partition $\nu$ such that $c_{\lambda \mu}^{\nu}>0$, we have $c_{\tilde{\lambda} \widetilde{\mu}}^{\nu}>0$.

Proof. It is known (see [7]) that $(I, J, K) \in \operatorname{LR}_{r}^{p}$ if and only if $\lambda(I), \lambda(J), \lambda(K)$ are the eigenvalues of some Hermitian matrices $X, Y$, and $Z=X+Y$. The claim then follows by Proposition 2.2 . 
Recall that a Littlewood-Richardson coefficient $c_{\lambda \mu}^{\nu}$ is the coefficient of the Schur function $s_{\nu}$ in the Schur function expansion of the product $s_{\lambda} s_{\mu}$. (For alternative representation-theoretic and intersection-theoretic interpretations, see, e.g., [6, 16.) In view of this, the assertion of Corollary 2.5 can be restated as follows.

Corollary 2.6. Among all ways to distribute the parts $\gamma_{1}, \gamma_{2}, \ldots$ of a given partition $\gamma=\left(\gamma_{1} \geq \gamma_{2} \geq \cdots\right)$ between two partitions $\lambda$ and $\mu$, there is one, namely, (17), for which the set of Schur functions contributing to the expansion of $s_{\lambda} s_{\mu}$ is largest by containment.

Conjecture 2.7. In Corollary [2.5, $c_{\widetilde{\lambda} \widetilde{\mu}}^{\nu} \geq c_{\lambda \mu}^{\nu}$. Thus, in Corollary [2.6, any expression of the form $s_{\tilde{\lambda}} s_{\widetilde{\mu}}-s_{\lambda} s_{\mu}$ is a nonnegative linear combination of Schur functions.

Example 2.8. Let $\gamma=(3,2,1)$. Then

$$
\begin{aligned}
s_{\widetilde{\lambda}} s_{\widetilde{\mu}}=s_{31} s_{2} & =s_{51}+s_{42}+s_{33}+s_{411}+s_{321}, \\
s_{32} s_{1} & =s_{42}+s_{33}+s_{321}, \\
s_{3} s_{21} & =s_{51}+s_{42}+s_{411}+s_{321} .
\end{aligned}
$$

We see that the right-hand side of (18) dominates each of the right-hand sides of (19)(20), in agreement with Corollary 2.6] and Conjecture 2.7]

Proposition 2.2 can be generalized to sums of several matrices, as follows.

Proposition 2.9. Let $\gamma_{1} \geq \cdots \geq \gamma_{m p}$ be the combined list of eigenvalues (with multiplicities) of $p$ by $p$ Hermitian matrices $A_{1}, \ldots, A_{m}$. Then there are Hermitian matrices $\widetilde{A}_{1}, \ldots, \widetilde{A}_{m}$ such that $\sum \widetilde{A}_{i}=\sum A_{i}$, and each $\widetilde{A}_{i}$ has eigenvalues $\gamma_{i}, \gamma_{i+m}, \ldots, \gamma_{i+m(p-1)}$.

Proof. This result is proved by combining Proposition 2.2 with an elementary combinatorial argument. Let us color the indexing set $\{1, \ldots, m p\}$ according to which eigenvalue comes from which matrix. More precisely, we use the colors $1, \ldots, m$, each of them exactly $p$ times, so that the following condition is satisfied:

(*) for each color $c$, the numbers $\gamma_{j}$ whose index $j$ has color $c$ are precisely the eigenvalues of $A_{c}$.

Consider the following "repainting" operation: pick two colors $c$ and $c^{\prime}$, identify the $2 p$ indices colored in these colors, and repaint these indices (if needed) so that their colors interlace as the indices increase: $c, c^{\prime}, c, c^{\prime}, \ldots$ (Alternatively, we may repaint them $\left.c^{\prime}, c, c^{\prime}, c, \ldots\right)$ The remaining $m p-2 p$ indices keep their colors. By Proposition 2.2, this operation can always be combined with an appropriate change of matrices $A_{c}$ and $A_{c^{\prime}}$ so that condition $(*)$ remains fulfilled and the sum $\sum A_{i}$ is unchanged.

To complete the proof, it suffices to show that any coloring of the set $\{1, \ldots, m p\}$ into colors $1, \ldots, m$ (using each color $p$ times) can be transformed by a sequence of repainting operations into the canonical coloring

$$
1, \ldots, m, 1, \ldots, m, \ldots \ldots, 1, \ldots, m
$$

where each index $j$ has color $c$ with $j \equiv c \bmod m$. Suppose we have a non-canonical coloring, and let $k$ be the smallest index whose color differs from the canonical one. 
Say, $k$ has color $c$, whereas in the canonical coloring, it has color $c^{\prime}$. By applying a repainting operation to the colors $c$ and $c^{\prime}$ we can change the color of $k$, thus expanding the initial segment colored in a canonical way. Iterating this procedure, we will arrive at the canonical coloring.

Using Proposition 2.9. one can extend Corollary 2.5 to $m$-tuples of partitions.

\section{Proof of Theorem 1.7}

We begin with a proposition that refines what it means for the Horn inequalities to hold. It is essentially equivalent to the main result of [8], improved by an idea from [13. We then use this proposition to give a quick proof of Theorem 1.7.

Let $a=\left(a_{1} \geq \cdots \geq a_{n}\right), b=\left(b_{1} \geq \cdots \geq b_{n}\right)$, and $c=\left(c_{1} \geq \cdots \geq c_{n}\right)$ be sequences of weakly decreasing real numbers of length $n$. For every $1 \leq r \leq n$, we have a collection of Horn inequalities

$$
\sum_{k \in K} c_{k} \leq \sum_{i \in I} a_{i}+\sum_{j \in J} b_{j},
$$

one for each $(I, J, K)$ in $\operatorname{LR}_{r}^{n}$. For $r=n$, there is just one inequality

$$
\sum_{k=1}^{n} c_{k} \leq \sum_{i=1}^{n} a_{i}+\sum_{j=1}^{n} b_{j} .
$$

Proposition 3.1. Assume that the sequences $a, b$, and $c$ consist of nonnegative real numbers. The following conditions are equivalent:

(i) The inequalities $\left(*_{r}^{n}\right)$ are satisfied for all $r \leq n$.

(ii) There are Hermitian $n$ by $n$ matrices $A, B$, and $C$ with eigenvalues $a_{1}, \ldots, a_{n}$, $b_{1}, \ldots, b_{n}$, and $c_{1}, \ldots, c_{n}$, respectively, such that $C \leq A+B$, i.e., $A+B-C$ is positive semidefinite.

(iii) For some integer $s \geq 1$, there are:

- real numbers $t(\ell) \in[0,1]$, for $1 \leq \ell \leq s$,

- a decomposition $n=\sum_{\ell=1}^{s} n(\ell)$ of $n$ into a sum of $s$ positive integers, and

- a decomposition of each of $a, b$, and $c$ into a union of $s$ subsequences, denoted $a(\ell), b(\ell)$, and $c(\ell)$, respectively, for $1 \leq \ell \leq s$, each of length $n(\ell)$,

such that the triples

$$
(t(\ell) \cdot a(\ell), t(\ell) \cdot b(\ell), c(\ell))
$$

satisfy all inequalities $\left(*_{r(\ell)}^{n(\ell)}\right)$ for $r(\ell) \leq n(\ell)$, with strict inequalities for $r(\ell)<$ $n(\ell)$, and equality for $r(\ell)=n(\ell)$.

(iv) For some integer $s \geq 1$, there are:

- real numbers $t(\ell) \in[0,1]$, for $1 \leq \ell \leq s$,

- a decomposition $n=\sum_{\ell=1}^{s} n(\ell)$ of $n$ into a sum of $s$ positive integers, and 
EIGENVALUES, SINGULAR VALUES, AND LITTLEWOOD-RICHARDSON COEFFICIENTS

- Hermitian $n(\ell)$ by $n(\ell)$ matrices $A(\ell), B(\ell)$, and $C(\ell)$, for $1 \leq \ell \leq s$, with

$$
C(\ell)=t(\ell)(A(\ell)+B(\ell))
$$

such that $A(\ell)$ and $B(\ell)$ preserve no proper subspace of $\mathbb{C}^{n(\ell)}$, and the eigenvalues of $\oplus A(\ell), \oplus B(\ell)$, and $\oplus C(\ell)$ are $a_{1}, \ldots, a_{n}, b_{1}, \ldots, b_{n}$, and $c_{1}, \ldots, c_{n}$, respectively.

Proof. The equivalence of (i) and (ii) is the main result of [8] (for three matrices).

(iii) $\Rightarrow$ (iv): By the Klyachko theorem [10] [7, Section 1], there are Hermitian matrices $A(\ell), B(\ell)$, and $C(\ell)$, with eigenvalues $a(\ell), b(\ell)$, and $c(\ell)$, such that $C(\ell)=t(\ell)(A(\ell)+$ $B(\ell)$ ). If there is any proper subspace $L$ preserved by $A(\ell)$ and $B(\ell)$, and therefore by $C(\ell)$, then $L^{\perp}$ is also preserved; after changing bases one can further decompose the matrices $A(\ell), B(\ell)$, and $C(\ell)$.

(iv) $\Rightarrow$ (ii): Take $A=\oplus A(\ell), B=\oplus B(\ell), C=\oplus C(\ell)$. Since $A(\ell)+B(\ell)$ is positive semidefinite, $C(\ell)=t(\ell)(A(\ell)+B(\ell)) \leq A(\ell)+B(\ell)$ for each $\ell$, so $C \leq A+B$.

(i) $\Rightarrow$ (iii): As in [13, let $t$ be the smallest real number such that the triple $(t \cdot a, t \cdot b, c)$ satisfies all inequalities $\left(*_{r}^{n}\right)$ for all $r \leq n$, but such that one (or more) of these inequalities holds with equality; let $(I, J, K) \in \overline{L R}_{r}^{n}$ be a triple for which equality holds. Decompose $a, b$, and $c$ respectively into subsequences given by

$$
\begin{aligned}
& a^{\prime}=\left(a_{i}\right)_{i \in I}, \quad b^{\prime}=\left(b_{j}\right)_{j \in J}, \quad c^{\prime}=\left(c_{k}\right)_{k \in K}, \\
& a^{\prime \prime}=\left(a_{i}\right)_{i \notin I}, \quad b^{\prime \prime}=\left(b_{j}\right)_{j \notin J}, \quad c^{\prime \prime}=\left(c_{k}\right)_{k \notin K} .
\end{aligned}
$$

By [8] (see the discussion after the statement of Theorem 2), there are $r$ by $r$ Hermitian matrices $A^{\prime}, B^{\prime}$, and $C^{\prime}$, with eigenvalues $a^{\prime}, b^{\prime}$, and $c^{\prime}$, with $C^{\prime}=t\left(A^{\prime}+B^{\prime}\right)$, and there are $n-r$ by $n-r$ matrices $A^{\prime \prime}, B^{\prime \prime}$, and $C^{\prime \prime}$, with eigenvalues $a^{\prime \prime}, b^{\prime \prime}$, and $c^{\prime \prime}$, with $C^{\prime \prime} \leq t\left(A^{\prime \prime}+B^{\prime \prime}\right)$. We may assume that $A^{\prime}$ and $B^{\prime}$ have no common invariant subspace, or they could be further decomposed. Take $t(1)=t, n(1)=r, A(1)=A^{\prime}, B(1)=B^{\prime}$, and $C(1)=C^{\prime}$. The triple $\left(t a^{\prime}, t b^{\prime}, c^{\prime}\right)$ satisfies the inequalities $*_{r}^{n}$ strictly for $r(1)<n(1)$, since any equality would lead to a proper invariant subspace by [7, Proposition 6 . The inductive hypothesis applies to the triple $\left(t \cdot a^{\prime \prime}, t \cdot b^{\prime \prime}, c^{\prime \prime}\right)$, and this produces the other terms in the required decomposition.

Remark 3.2. Even if all the inequalities $\left(*_{r}^{n}\right)$ for $r<n$ are strict for a particular triple $(a, b, c)$, this does not imply that the triple is indecomposable (in the sense of (iii)). For example, the triple $((2,1,0),(2,1,0),(3,2,1))$ satisfies all inequalities $\left(*_{r}^{3}\right)$ strictly for $r<3$, with equality for $r=3$; but it decomposes into the three triples $((2),(1),(3))$, $((1),(0),(1))$, and $((0),(2),(2))$. Note also that decompositions need not be unique, as this triple also decomposes into $((1),(2),(3)),((0),(1),(1))$, and $((2),(0),(2))$.

Remark 3.3. Proposition 3.1 extends as usual (cf. [7, 8) to the case where $a$ and $b$ are replaced by any number $m \geq 2$ of sequences, and with $m$ matrices in place of $A$ and $B$. Furthermore, the Hermitian matrices in (ii) and (iv) can be taken to be real symmetric. (They may have no real invariant subspaces, even if they have complex invariant subspaces, but (iv) is true with either interpretation.) 
We are now ready to prove Theorem 1.7

Let us first check that the equivalences (ii) $\Leftrightarrow($ iii $) \Leftrightarrow$ (iv) in Theorem 1.7 follow from 8 . The equivalence of (ii) and (iii) is the result of 8 , while (iii) $\Rightarrow$ (iv) is obvious.

(iv) $\Rightarrow$ (ii): Let the eigenvalues of $A$ and $B$ be $a_{1}, \ldots, a_{p}$ and $b_{1}, \ldots, b_{p}$. The inequality $2 C \leq A-B$ gives the inequalities

$$
2 \sum_{k \in K} s_{k} \leq \sum_{i \in I} a_{i}-\sum_{j \in J} b_{p+1-j}
$$

for all $(I, J, K) \in \mathrm{LR}_{r}^{p}, r \leq p$. Since $a_{1} \geq \cdots \geq a_{p}$ and $b_{1} \geq \cdots \geq b_{p}$ are subsequences of $\lambda_{1} \geq \ldots \lambda_{2} \geq \ldots \geq \lambda_{n}$, it follows that $\lambda_{i} \geq a_{i}$ and $\lambda_{n+1-i} \leq b_{p+1-i}$ for $1 \leq i \leq p$. Hence

$$
\sum_{i \in I} a_{i}-\sum_{j \in J} b_{p+1-j} \leq \sum_{i \in I} \lambda_{i}-\sum_{j \in J} \lambda_{n+1-j},
$$

and (ii) follows.

To prove the equivalence of (i) and (ii), we use Proposition 3.1. Note that both conditions (and in fact each of (i)-(iv)) is unchanged if every $\lambda_{i}$ is replaced by $\lambda_{i}+c$, for any real number $c$. This is obvious in (ii), and follows by adding a scalar matrix $c I_{n}$ to the matrix in (i). Hence we may assume that $\lambda_{p} \geq 0 \geq \lambda_{n+1-p}$. Then all three sequences

$$
\left(\lambda_{1} \geq \cdots \geq \lambda_{p}\right), \quad\left(-\lambda_{n} \geq \cdots \geq-\lambda_{n+1-p}\right), \quad\left(2 s_{1} \geq \cdots \geq 2 s_{p}\right)
$$

consist of nonnegative numbers, so Proposition 3.1 applies to them.

(ii) $\Rightarrow$ (i): We use a decomposition as in Proposition [3.1(iv), but with $n$ replaced by $p$. This produces a decomposition $p=\sum p(\ell)$, numbers $t(\ell) \in[0,1]$, and $p(\ell)$ by $p(\ell)$ Hermitian matrices $A(\ell), B(\ell)$, and $C(\ell)$ such that $2 C(\ell)=t(\ell)(A(\ell)-B(\ell))$, the eigenvalues of $\oplus A(\ell)$ are $\lambda_{1}, \ldots, \lambda_{p}$, the eigenvalues of $\oplus B(\ell)$ are $\lambda_{n+1-p}, \ldots, \lambda_{n}$, and the eigenvalues of $\oplus C(\ell)$ are $s_{1}, \ldots, s_{p}$. Following [13], for each $\ell$, choose $\theta(\ell)$ so that $\sin (2 \theta(\ell))=t(\ell)$. Writing matrices in block form, define $p(\ell)$ by $p(\ell)$ Hermitian (or real symmetric) matrices $P(\ell)$ and $Q(\ell)$ by the identities

$$
\left[\begin{array}{cc}
P(\ell) & C(\ell) \\
C(\ell)^{*} & Q(\ell)
\end{array}\right]=\left[\begin{array}{cc}
\cos (\theta(\ell)) & -\sin (\theta(\ell)) \\
\sin (\theta(\ell)) & \cos (\theta(\ell))
\end{array}\right] \cdot\left[\begin{array}{cc}
A(\ell) & 0 \\
0 & B(\ell)
\end{array}\right] \cdot\left[\begin{array}{cc}
\cos (\theta(\ell)) & \sin (\theta(\ell)) \\
-\sin (\theta(\ell)) & \cos (\theta(\ell))
\end{array}\right] .
$$

The direct sum of the matrices $\left[\begin{array}{cc}P(\ell) & C(\ell) \\ C(\ell)^{*} & Q(\ell)\end{array}\right]$, together with the diagonal matrix of size $n-2 p$ with diagonal entries $\lambda_{p+1}, \ldots, \lambda_{n-p}$ has eigenvalues $\lambda_{1}, \ldots, \lambda_{n}$. The direct sum $X$ of the matrices $C(\ell)$, filled in with 0's on the right, is the upper $p$ by $n-p$ block $X$ of this matrix, and its singular values are $s_{1}, \ldots, s_{p}$.

(i) $\Rightarrow$ (ii): The argument is similar to the proof of Proposition 1.16, Let $Z=\left[\begin{array}{c}P \\ X^{*}\end{array} \underset{Q}{X}\right]$ be a matrix as in (i). The matrix $\widetilde{Z}=\left[\begin{array}{cc}-P & X \\ X^{*} & -Q\end{array}\right]$ has eigenvalues $-\lambda_{n}, \ldots,-\lambda_{1}$, as follows from the identity (3) , with $U_{1}=\sqrt{-1} I_{p}, V_{1}=U_{1}^{*}=-\sqrt{-1} I_{p}, U_{2}=-\sqrt{-1} I_{n-p}$, and $V_{2}=U_{2}^{*}=\sqrt{-1} I_{n-p}$. Furthermore, $Z+\widetilde{Z}=\left[\begin{array}{cc}0 \\ 2 X^{*} & 0\end{array}\right]$ has eigenvalues

$$
2 s_{1} \geq \ldots 2 s_{p} \geq 0 \geq \ldots \geq 0 \geq-2 s_{p} \geq \ldots \geq-2 s_{1} .
$$


By the Horn inequalities for sums of Hermitian matrices (see [7, $\S 1$ ), we have, for all $(I, J, K)$ in $\mathrm{LR}_{r}^{p} \subset \mathrm{LR}_{r}^{n}, r \leq p$

$$
\sum_{k \in K} 2 s_{k} \leq \sum_{i \in I} \lambda_{i}+\sum_{j \in J}\left(-\lambda_{n+1-j}\right)
$$

which is the assertion in (ii).

That $X$ can be specified in advance follows as in Remark 1.6. That the Hermitian matrices can be taken to be real symmetric follows from the analogous results in [7, 8].

\section{2-QUOTIENTS AND LITTLEWOOD-RICHARDSON COEFFICIENTS}

4.1. The 2-quotient correspondence. The material reviewed in this section goes back to T. Nakayama 14. (in a somewhat different language). For detailed exposition and further references, see, e.g., [16], Exercise 7.59 and its solution, or [5].

Definition 4.1. For two sets $I=\left\{i_{1}<i_{2}<\ldots<i_{r}\right\}$ and $J=\left\{j_{1}<j_{2}<\ldots<j_{r}\right\}$ of positive integers, define (cf. equation (12))

$$
\tau(I, J)=\left\{2 i_{1}-1, \ldots, 2 i_{r}-1\right\} \cup\left\{2 j_{1}, \ldots, 2 j_{r}\right\} .
$$

It is easy to check that the corresponding map on partitions is well defined. To be more precise, let $\lambda=\left(\lambda_{1}, \lambda_{2}, \ldots\right)$ and $\mu=\left(\mu_{1}, \mu_{2}, \ldots\right)$ be two integer partitions, let $\ell(\lambda)$ (resp., $\ell(\mu)$ ) be the number of nonzero parts in $\lambda$ (resp., in $\mu$ ), and let $r \geq \max (\ell(\lambda), \ell(\mu)$ ). Then there are uniquely defined $r$-element sets of positive integers $I$ and $J$ that correspond to $\lambda$ and $\mu$, respectively, under the map (11). Furthermore, the partition $\tau(\lambda, \mu)$ that corresponds to $\tau(I, J)$ under (11) only depends on the partitions $\lambda$ and $\mu$ and not on the sets $I$ and $J$ (that is, not on the choice of $r$ ). If one traces the Young diagram of a partition by a sequence of horizontal and vertical steps, moving from Southwest to Northeast in a rectangle containing the diagrams of $\lambda$ and $\mu$, the diagram of $\tau(\lambda, \mu)$ is traced, in a rectangle twice as wide in both directions, by alternating steps from $\lambda$ and $\mu$, starting with the first step of $\lambda$.

It is easy to check that $|\tau(\lambda, \mu)|=2(|\lambda|+|\mu|)$, where we use the notation $|\lambda|=\sum \lambda_{i}$.

Example 4.2. Let $\lambda=(2,1)$ and $\mu=(2)$. Taking $r=2$ gives $I=\{2,4\}, J=\{1,4\}$, $\tau(I, J)=\{2,3,7,8\}$, and finally $\tau(\lambda, \mu)=(4,4,1,1)$. On the other hand, $r=3$ yields $I=\{1,3,5\}, J=\{1,2,5\}, \tau(I, J)=\{1,2,4,5,9,10\}$, and again $\tau(\lambda, \mu)=(4,4,1,1)$.

We identify each partition $\lambda=\left(\lambda_{1}, \lambda_{2}, \ldots\right)$ with the Young diagram that represents it (i.e., the one with row lengths $\lambda_{1}, \lambda_{2}, \ldots$ ). In this language, Example 4.2 becomes

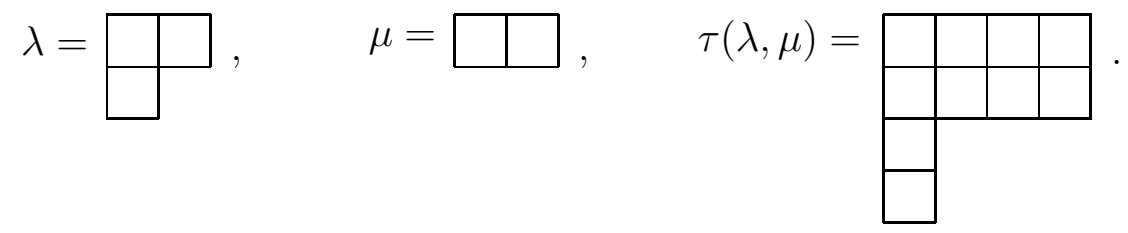

Example 4.3. In the special case $\lambda=\mu=\left(\lambda_{1}, \lambda_{2}, \ldots\right)$, one easily verifies that

$$
\tau(\lambda, \lambda)=\left(2 \lambda_{1}, 2 \lambda_{1}, 2 \lambda_{2}, 2 \lambda_{2}, \ldots\right) .
$$


That is, $\tau(\lambda, \lambda)$ is obtained from $\lambda$ by a dilation with coefficient 2 (in both directions).

A Young diagram is called domino-decomposable if it can be partitioned into disjoint $1 \times 2$ rectangles (dominoes). The following result is a special case of a theorem of T. Nakayama [14].

Proposition 4.4. The Young diagram $\tau(\lambda, \mu)$ is always domino-decomposable. The map $(\lambda, \mu) \mapsto \tau(\lambda, \mu)$ is a bijection between ordered pairs of partitions (or Young diagrams), on one hand, and domino-decomposable Young diagrams, on another.

The pair of partitions $(\lambda, \mu)$ that corresponds to a given domino-decomposable Young diagram $\tau=\tau(\lambda, \mu)$ is traditionally called the 2-quotient of $\tau$.

In the notation introduced above, Proposition 1.19 is equivalent to the following:

Proposition 4.5. For any domino-decomposable Young diagram $\tau$ with 2-quotient $(\lambda, \mu)$, and for any Young diagram $\nu$ with $|\nu|=|\lambda|+|\mu|$, we have $c_{\lambda, \mu}^{\nu} \leq c_{\tau(\lambda, \mu), \tau(\lambda, \mu)}^{\tau(\nu, \nu)}$.

4.2. The result of Carré and Leclerc. The proof of Proposition 4.5 is based on the version of the Littlewood-Richardson Rule due to Carré and Leclerc [3] (see Proposition 4.6 below), which expresses a Littlewood-Richardson coefficient $c_{\lambda \mu}^{\nu}$ as the number of "domino tableaux" satisfying certain conditions. We briefly review this result here, referring the reader to [3] or [12] for fine-print technicalities.

A (semistandard) domino tableau $T$ of shape $\tau$ consists of a decomposition of $\tau$ into dominoes together with the labelling of each domino by a positive integer. The labelling must satisfy two conditions analogous to the usual conditions imposed on (semistandard) Young tableaux: the labels weakly increase in rows and strictly increase in columns.

The weight of $T$ is the sequence $\nu=\left(\nu_{1}, \nu_{2}, \ldots\right)$ in which each entry $\nu_{i}$ is equal to the number of labels in $T$ equal to $i$. The reading word $w(T)$ is obtained by scanning the labels of $T$ column by column, right to left and top down. To clarify, when we read a tableau by columns, right-to-left, an entry in a horizontal domino is skipped the first time we trace it. (Carré and Leclerc use the French notation, with the tableau flipped upside down with respect to our conventions, and their reading order is reverse to ours.)

A domino tableau $T$ is called a Yamanouchi domino tableaux (YDT) if it satisfies the following additional restriction: its reading word $w(T)$ is a Yamanouchi word, or a lattice permutation (see [16, page 432]), that is,

(21) every entry $i$ appears in any initial segment of $w(T)$ at least as many times as any entry $j>i$.

Figure 1 lists all YDT of shape $(4,4,1,1)$, their respective reading words, and weights.

Proposition 4.6. [3. Corollary 4.4] A Littlewood-Richardson coefficient $c_{\lambda \mu}^{\nu}$ is equal to the number of Yamanouchi domino tableaux of shape $\tau(\lambda, \mu)$ and weight $\nu$.

To illustrate, let $\lambda=(2,1)$ and $\mu=(2)$ (see Example 4.2). Then $\tau(\lambda, \mu)=(4,4,1,1)$. Comparing Proposition 4.6 with Figure 1, we conclude that there are 4 nonvanishing 


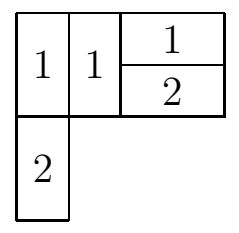

12112 $(3,2)$

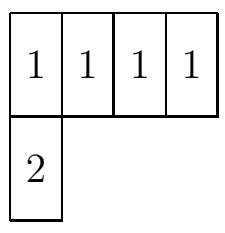

11112

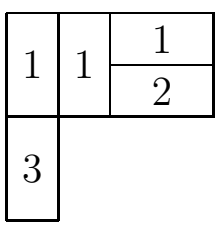

12113

$(3,1,1)$

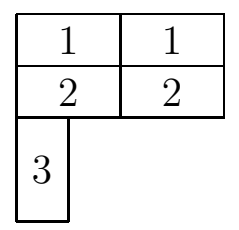

12123

$(2,2,1)$

Figure 1. Yamanouchi domino tableaux of shape $(4,4,1,1)$

Littlewood-Richardson coefficients $c_{\lambda \mu}^{\nu}$, all equal to 1:

$$
c_{21,2}^{32}=c_{21,2}^{41}=c_{21,2}^{311}=c_{21,2}^{221}=1 .
$$

Accordingly, the Schur functions $s_{\lambda}$ and $s_{\mu}$ multiply as follows:

$$
s_{21} s_{2}=s_{32}+s_{41}+s_{311}+s_{221} .
$$

We note that a direct link between Proposition 4.6 and the traditional versions of the Littlewood-Richardson Rule was established by M. A. A. van Leeuwen [12].

4.3. Proof of Proposition 4.5. Set $\rho=\tau(\lambda, \mu)$. We need to show that $c_{\lambda \mu}^{\nu} \leq c_{\rho \rho}^{\tau(\nu, \nu)}$. By Proposition 4.6, these Littlewood-Richardson coefficients are given by

$$
\begin{aligned}
c_{\lambda \mu}^{\nu} & =\text { number of YDT of shape } \rho \text { and weight } \nu, \\
c_{\rho, \rho}^{\tau(\nu, \nu)} & =\text { number of YDT of shape } \tau(\rho, \rho) \text { and weight } \tau(\nu, \nu) .
\end{aligned}
$$

Recall from Example 4.3 that $\tau(\rho, \rho)$ and $\tau(\nu, \nu)$ are obtained from $\rho$ and $\nu$, respectively, by a dilation with coefficient 2 . To prove the inequality, we need an injection $T \mapsto T^{\prime}$ from the first set of YDT to the second one. To construct such an injection, simply chop each domino (say, with a label $k$ ) in a YDT $T$ of shape $\rho$ and weight $\nu$ into 4 quarter-size dominoes; then put the labels $2 k-1$ into the top two dominoes, and $2 k$ into the bottom two. To illustrate, the leftmost tableau $T$ in Figure 1 will transform as shown in Figure 2

We then need to check that

(i) the resulting tableau $T^{\prime}$ is a valid (semistandard) domino tableau;

(ii) $T^{\prime}$ has shape $\tau(\rho, \rho)$ and weight $\tau(\nu, \nu)$;

(iii) $T^{\prime}$ satisfies the Yamanouchi condition (21) for any $i$ and $j=i+1$.

Verifying the claims (i) and (ii) is straightforward. Claim (iii), for $i$ odd, is also easy: each entry $i+1$ is preceded by $i$ in the reading word $w\left(T^{\prime}\right)$. The case of $i$ even requires careful examination of a handful of cases. For $i=2 k$, we need to look at a domino labelled $k+1$ in the original tableau $T$ (see Figure 3) and check that each of the corresponding entries equal to $2 k+1$ in $T^{\prime}$ (marked by a bullet $\bullet$ in Figure 3) appears in the reading word $w\left(T^{\prime}\right)$ at the end of an initial segment that contains more $2 k$ 's than $2 k+1$ 's. This can 
be done by looking at all dominoes labeled $k$ or $k+1$ in the shaded region in Figure 3, and invoking condition (21) for the tableau $T$. The details are left to the reader.

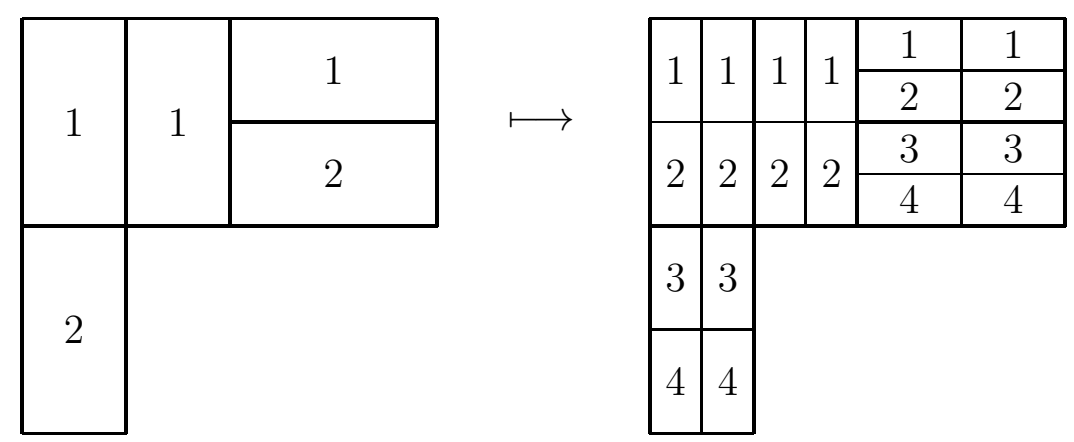

FiguRE 2. Injection $T \mapsto T^{\prime}$ in the proof of Proposition 4.5
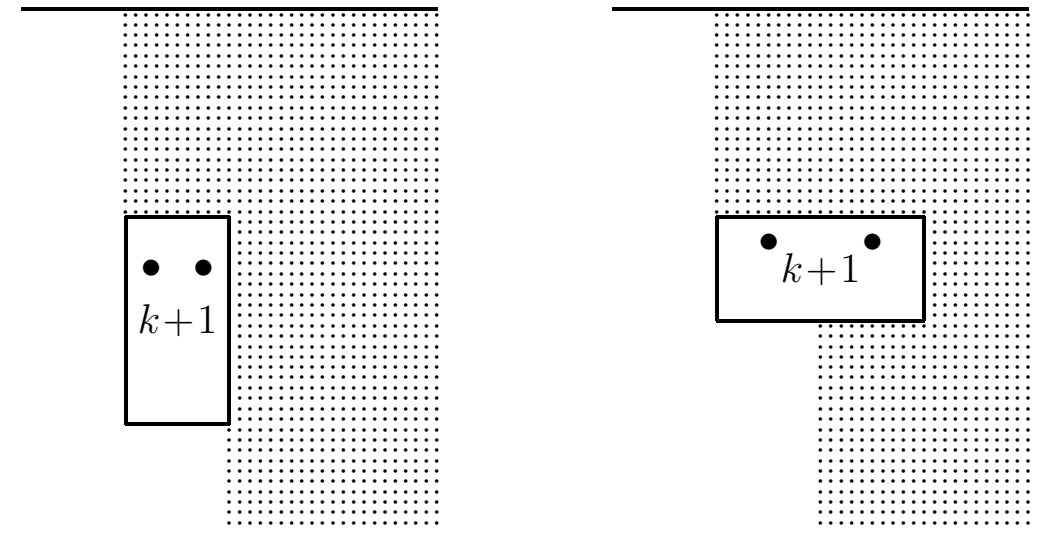

Figure 3. Checking condition (iii)

\section{Grassmann geometry}

In this section we sketch a geometric approach to the proof of Proposition 4.5 Carrying this out leads to another problem about Littlewood-Richardson coefficients - but this remains a conjecture. We begin by stating the conjecture, which does not require any geometry.

5.1. A combinatorial conjecture. Given an ordered pair $(\lambda, \mu)$ of partitions with the same number of parts, define a new ordered pair $\left(\lambda^{*}, \mu^{*}\right)$ by the following recipe:

$$
\begin{aligned}
& \lambda_{k}^{*} \stackrel{\text { def }}{=} \lambda_{k}-k+\#\left\{\ell \mid \mu_{\ell}-\ell \geq \lambda_{k}-k\right\} ; \\
& \mu_{\ell}^{*} \stackrel{\text { def }}{=} \mu_{\ell}-\ell+1+\#\left\{k \mid \lambda_{k}-k>\mu_{\ell}-\ell\right\} .
\end{aligned}
$$


For example, if $\lambda=(5,5,2,2)$ and $\mu=(1,1,0,0)$, then $\lambda^{*}=(4,3,1,0)$ and $\mu^{*}=(3,2,2,1)$.

We say that a partition fits in a $p$ by $n-p$ rectangle if it has at most $p$ positive parts, each of size at most $n-p$. Let $\lambda$ and $\mu$ be such partitions, and let $I$ and $J$ be the $p$-element subsets of the set $\{1, \ldots, n\}$ associated to $\lambda$ and $\mu$, respectively, under the correspondence (11). Then the sets $I^{*}$ and $J^{*}$ associated to $\lambda^{*}$ and $\mu^{*}$ are defined by

$$
\begin{aligned}
& I^{*}=\left\{i+\#\left\{i^{\prime} \in I \mid i^{\prime}<i\right\}-\#\{j \in J \mid j<i\}\right\}_{i \in I}, \\
& J^{*}=\left\{j+\#\left\{j^{\prime} \in J \mid j^{\prime} \leq j\right\}-\#\{i \in I \mid i \leq j\}\right\}_{j \in J} .
\end{aligned}
$$

Using this reformulation of the transformation $(\lambda, \mu) \mapsto\left(\lambda^{*}, \mu^{*}\right)$, it is easy to verify that $\lambda^{*}$ and $\mu^{*}$ are partitions, that both of them fit in a $p$ by $n-p$ rectangle, and $\left|\lambda^{*}\right|+\left|\mu^{*}\right|=|\lambda|+|\mu|$.

Conjecture 5.1. For any partition $\nu$, we have $c_{\lambda \mu}^{\nu} \leq c_{\lambda^{*} \mu^{*}}^{\nu}$.

Equivalently, in terms of Schur functions, $s_{\lambda^{*}} s_{\mu^{*}}-s_{\lambda} s_{\mu}$ is Schur positive, i.e., when this difference is expressed as a linear combination of Schur functions $s_{\nu}$, all the coefficients are nonnegative.

Using [2], A. Buch has verified this conjecture for all pairs $(\lambda, \mu)$ where both $\lambda$ and $\mu$ fit in a $p$ by $q$ rectangle with $p q \leq 48$.

A pair of partitions $(\lambda, \mu)$ is a fixed point of the operation $*$ if and only if the sequence $\mu_{1}, \lambda_{1}, \mu_{2}, \lambda_{2}, \mu_{3}, \ldots$ is weakly decreasing. In fact, for any other $(\lambda, \mu)$, if $k=k(\lambda, \mu)$ is maximal such that the first $k$ terms of this sequence form a weakly decreasing sequence, then the corresponding sequence for $\left(\lambda^{*}, \mu^{*}\right)$ has the same first $k-1$ terms as that for $(\lambda, \mu)$, while its $k^{\text {th }}$ term is strictly larger; and $k\left(\lambda^{*}, \mu^{*}\right) \geq k(\lambda, \mu)$. From this it follows that, after applying the $*$ operation a finite number of times, one always reaches a fixed point - a fact which is also an easy consequence of the conjecture.

5.2. Intersections of Schubert cells. In the rest of Section 5, we present a geometric argument showing how Conjecture 5.1 implies Proposition 4.5. The general shape of the argument is as follows. First, we formulate a geometric conjecture (see Conjecture 5.3) asserting transversality of certain intersections of Schubert cells, and explain why this transversality conjecture would imply Proposition 4.5. We then derive the transversality conjecture from Conjecture 5.1 by an analysis of tangent spaces, using a result of P. Belkale.

We begin by recalling the basic facts of the Schubert calculus on Grassmannians, while setting up the relevant notation. (See [6] for further details.) Let $V$ be an $n$-dimensional vector space over an algebraically closed field. For any complete flag

$$
E_{\bullet}=\left(0=E_{0} \subset E_{1} \subset \cdots \subset E_{n}=V\right)
$$

of subspaces of $V$, and any partition $\lambda$ whose Young diagram fits in a $p$ by $n-p$ rectangle, there is a Schubert variety $\Omega_{\lambda}\left(E_{\bullet}\right)$ in the $\operatorname{Grassmannian} \operatorname{Gr}(p, V)$ of $p$-dimensional 
subspaces of $V$, defined by

$$
\Omega_{\lambda}\left(E_{\bullet}\right)=\left\{L \in \operatorname{Gr}(p, V) \mid \operatorname{dim}\left(L \cap E_{n-p+k-\lambda_{k}}\right) \geq k \text { for } 1 \leq k \leq p\right\} .
$$

This is the closure of the corresponding Schubert cell $\Omega_{\lambda}^{\circ}\left(E_{\bullet}\right)$, which consists of all subspaces $L \in \operatorname{Gr}(p, V)$ such that, for $0 \leq m \leq n$ and $0 \leq k \leq p$, we have $\operatorname{dim}\left(L \cap E_{m}\right)=k$ if and only if

$$
n-p+k-\lambda_{k} \leq m \leq n-p+k-\lambda_{k+1} .
$$

If $I \subset\{1, \ldots, n\}$ corresponds to $\lambda$ as in (11), then

$$
\Omega_{\lambda}^{\circ}\left(E_{\bullet}\right)=\left\{L \in \operatorname{Gr}(p, V) \mid \operatorname{dim}\left(L \cap E_{m}\right)=\#\{i \in I \mid i>n-m\} \text { for any } m\right\} .
$$

The Schubert cell $\Omega_{\lambda}^{\circ}\left(E_{\bullet}\right)$ is a manifold (isomorphic to an affine space) of codimension $|\lambda|$ in $\operatorname{Gr}(p, V)$.

Let $\lambda, \mu$, and $\nu$ be partitions whose Young diagrams fit in a $p$ by $n-p$ rectangle, and assume that the Littlewood-Richardson number $c_{\lambda \mu \nu} \stackrel{\text { def }}{=} c_{\lambda \mu}^{\nu^{\vee}}$ is positive, where we set $\nu^{\vee}=\left(n-p-\nu_{p}, \ldots, n-p-\nu_{1}\right)$. Take three complete flags $E_{\bullet}, F_{\bullet}, G_{\bullet}$ in $V=\mathbb{C}^{n}$ which are in general position. The latter assumption implies that the corresponding Schubert cells $\Omega_{\lambda}^{\circ}\left(E_{\bullet}\right), \Omega_{\mu}^{\circ}\left(F_{\bullet}\right), \Omega_{\nu}^{\circ}\left(G_{\bullet}\right)$ meet transversally in $c_{\lambda \mu \nu}$ points, i.e., there are $c_{\lambda \mu \nu}$ subspaces $L$ of dimension $p$ in $V$ that are in the transversal intersection of these Schubert cells.

We next construct three complete flags $A_{\bullet}, B_{\bullet}$, and $C_{\bullet}$ in the $2 n$-dimensional vector space $V \oplus V$, by setting, for $1 \leq m \leq n$,

$$
\begin{aligned}
& A_{2 m}=F_{m} \oplus E_{m}, \quad A_{2 m-1}=F_{m-1} \oplus E_{m}, \\
& B_{2 m}=E_{m} \oplus F_{m}, \quad B_{2 m-1}=E_{m} \oplus F_{m-1} \text {, } \\
& C_{2 m}=G_{m} \oplus G_{m}, \quad C_{2 m-1}=G_{m} \oplus G_{m-1} .
\end{aligned}
$$

Lemma 5.2. If $L \in \Omega_{\lambda}^{\circ}\left(E_{\bullet}\right) \cap \Omega_{\mu}^{\circ}\left(F_{\bullet}\right) \cap \Omega_{\nu}^{\circ}\left(G_{\bullet}\right)$, then

$$
L \oplus L \in \Omega_{\tau(\lambda, \mu)}^{\circ}\left(A_{\bullet}\right) \cap \Omega_{\tau(\lambda, \mu)}^{\circ}\left(B_{\bullet}\right) \cap \Omega_{\tau(\nu, \nu)}^{\circ}\left(C_{\bullet}\right) .
$$

Proof. This is a straightforward verification based on the definitions (12) and (23); e.g.,

$$
\begin{aligned}
& \operatorname{dim}\left((L \oplus L) \cap A_{2 m}\right) \\
= & \operatorname{dim}\left(L \cap F_{m}\right)+\operatorname{dim}\left(L \cap E_{m}\right) \\
= & \#\{i \in I \mid i>n-m\}+\#\{j \in J \mid j>n-m\} \\
= & \#\{i \in \tau(I, J) \mid i>2 n-2 m\},
\end{aligned}
$$

as desired; here $J$ and $\tau(I, J)$ denote the subsets that correspond to $\mu$ and $\tau(\lambda, \mu)$, respectively.

Conjecture 5.3. In Lemma 5.2, the intersection of Schubert cells appearing in (25) is transversal at each such point $L \oplus L$.

Remark 5.4. The transversality would be automatic if the flags $A_{\bullet}, B_{\bullet}$, and $C_{\bullet}$ were in general position with respect to each other; but they are not, already for $n=1$. 
Conjecture [5.3, or the weaker assertion that these three cells intersect properly at each such point (i.e., $L \oplus L$ is an isolated point of the intersection) is enough to imply that

$$
c_{\lambda, \mu, \nu} \leq c_{\tau(\lambda, \mu), \tau(\lambda, \mu), \tau(\nu, \nu)},
$$

which is equivalent to Proposition 4.5. (Here we are using the fact that, even if this intersection of Schubert varieties should contain connected components of positive dimension, their contribution to the total intersection number must be nonnegative; this follows, e.g., from the fact that the tangent bundle of the Grassmannian is generated by its global sections.)

5.3. Conjecture 5.1 implies Conjecture5.3. Recall that the tangent space to $\operatorname{Gr}(p, V)$ at a point $L$ is canonically identified with $\operatorname{Hom}(L, V / L)$.

We use some basic facts about tangent spaces to Schubert cells, which can be found in Belkale's preprint [1]. If $L$ is in a Schubert cell $\Omega_{\lambda}^{\circ}\left(E_{\bullet}\right)$, then its tangent space $T_{[L]}\left(\Omega_{\lambda}^{\circ}\left(E_{\bullet}\right)\right)$ is the subspace of $\operatorname{Hom}(L, V / L)$ consisting of linear maps $\phi$ such that

$$
\phi\left(E_{m} \cap L\right) \subset\left(L+E_{m}\right) / L
$$

for all $m$. These intersections $E_{m} \cap L$ and quotients $\left(L+E_{m}\right) / L$ give general flags $E_{\text {. }}^{\prime}$ and $E_{\bullet}^{\prime \prime}$ in $L$ and $V / L$, respectively. More precisely (cf. (22) ), the flag $E_{\bullet}^{\prime}$ in $L$ is given by

$$
E_{k}^{\prime}=E_{n-p+k-\lambda_{k}} \cap L
$$

for $1 \leq k \leq p$, and the flag $E_{\bullet}^{\prime \prime}$ in $V / L$ consists of the spaces $\left(L+E_{m}\right) / L$, for all $m$ not of the form $n-p+k-\lambda_{k}$. An equivalent condition on the tangent space (which we will also use) is that a map $\phi \in T_{[L]}\left(\Omega_{\lambda}^{\circ}\left(E_{\bullet}\right)\right)$ sends $E_{k}^{\prime}$ to $E_{n-p-\lambda_{k}}^{\prime \prime}$, for $1 \leq k \leq p$. Summarizing,

$$
\begin{aligned}
T_{[L]}\left(\Omega_{\lambda}^{\circ}\left(E_{\bullet}\right)\right) & =\left\{\phi: L \rightarrow V / L \mid \phi\left(E_{m} \cap L\right) \subset\left(L+E_{m}\right) / L, \text { for } 1 \leq m \leq n\right\} \\
& =\left\{\phi: L \rightarrow V / L \mid \phi\left(E_{k}^{\prime}\right) \subset E_{n-p-\lambda_{k}}^{\prime \prime}, \text { for } 1 \leq k \leq p\right\} .
\end{aligned}
$$

Proposition 5.5. 1] Assume $|\lambda|+|\mu|+|\nu|=p(n-p)$. The following are equivalent:

(i) $c_{\lambda \mu \nu}>0$.

(ii) For any general complete flags $P_{\bullet}, Q_{\bullet}$, and $R_{\bullet}$ in a p-dimensional vector space $L$, and $P_{\bullet}^{\prime}, Q_{\bullet}^{\prime}$, and $R_{\bullet}^{\prime}$ in an $(n-p)$-dimensional vector space $L^{\prime}$, if $\phi: L \rightarrow L^{\prime}$ is a linear map that sends $P_{i}$ to $P_{n-p-\lambda_{i}}^{\prime}, Q_{i}$ to $Q_{n-p-\mu_{i}}^{\prime}$, and $R_{i}$ to $R_{n-p-\nu_{i}}^{\prime}$, then $\phi=0$.

Recall that we are working under the assumptions that

- $c_{\lambda \mu \nu}>0$;

- $E_{\bullet}, F_{\bullet}, G_{\bullet}$ are three complete flags in $V=\mathbb{C}^{n}$ in general position;

- $L$ lies in the intersection of the Schubert cells $\Omega_{\lambda}^{\circ}\left(E_{\bullet}\right), \Omega_{\mu}^{\circ}\left(F_{\bullet}\right), \Omega_{\nu}^{\circ}\left(G_{\bullet}\right)$.

To illustrate Proposition 5.5, let us apply it to the flags induced on $L$ and $V / L$ by $E_{\bullet}$, $F_{\bullet}$, and $G_{\bullet}$ (cf. (27) ); we then recover the transversality of the intersection at $L$. 
Recall the definition (24) of the flags $A_{\bullet}, B_{\bullet}$, and $C_{\bullet}$ in $V \oplus V$. Conjecture 5.3 says that for every $L$ as above, any linear map $\Phi: L \oplus L \rightarrow(V \oplus V) /(L \oplus L)$ that satisfies

$$
\begin{aligned}
& \Phi\left(A_{k} \cap(L \oplus L)\right) \subset\left((L \oplus L)+A_{k}\right) /(L \oplus L), \\
& \Phi\left(B_{k} \cap(L \oplus L)\right) \subset\left((L \oplus L)+B_{k}\right) /(L \oplus L), \\
& \Phi\left(C_{k} \cap(L \oplus L)\right) \subset\left((L \oplus L)+C_{k}\right) /(L \oplus L),
\end{aligned}
$$

for all $1 \leq k \leq 2 n$, must be the zero map. Regarding $\Phi$ as a 2 by 2 matrix $\Phi=\left[\begin{array}{ll}\phi_{11} & \phi_{12} \\ \phi_{21} & \phi_{22}\end{array}\right]$ of maps from $L$ to $V / L$, one can restate conditions (28) as saying that both $\phi_{11}$ and $\phi_{22}$ satisfy

$$
\begin{aligned}
& \phi\left(E_{m} \cap L\right) \subset\left(L+E_{m}\right) / L, \\
& \phi\left(F_{m} \cap L\right) \subset\left(L+F_{m}\right) / L, \\
& \phi\left(G_{m} \cap L\right) \subset\left(L+G_{m}\right) / L,
\end{aligned}
$$

while both $\phi_{12}$ and $\phi_{21}$ satisfy

$$
\begin{aligned}
& \phi\left(E_{m} \cap L\right) \subset\left(L+F_{m}\right) / L, \\
& \phi\left(F_{m} \cap L\right) \subset\left(L+E_{m-1}\right) / L, \\
& \phi\left(G_{m} \cap L\right) \subset\left(L+G_{m}\right) / L,
\end{aligned}
$$

for $1 \leq m \leq n$. The transversality of the three original Schubert cells implies that any $\phi$ satisfying (29) is the zero map, so the two diagonal maps vanish. It remains to show that the two off-diagonal maps vanish as well. We will deduce it from (30)-(32) using Proposition 5.5 and Conjecture 5.1 .

Let us apply Proposition 5.5 to the situation where

- the flags $P_{\bullet}, Q_{\bullet}, R_{\bullet}$ are induced on $L$ by $E_{\bullet}, F_{\bullet}, G_{\bullet}$, respectively;

- $P_{\bullet}^{\prime}, Q_{\bullet}^{\prime}, R_{\bullet}^{\prime}$ are induced on $L^{\prime}=V / L$ by $F_{\bullet}, E_{\bullet}, G_{\bullet}$, respectively (in this order!);

- the three partitions are $\lambda^{*}, \mu^{*}$, and $\nu$.

By Conjecture [5.1], we have $c_{\lambda^{*} \mu^{*} \nu}>0$, so Proposition 5.5]applies. We claim that unraveling condition (ii) of Proposition 5.5 in this particular situation, one obtains precisely that (30)- (32) implies $\phi=0$, as needed. To explain why, we will demonstrate how (30) translates into $\phi\left(E_{k}^{\prime}\right) \subset F_{n-p-\lambda_{k}^{*}}^{\prime \prime}$, where we are using the same notation as in the sentence containing (26). (The checks involving (31) and (32) are similar.) It follows from the definition of a Schubert cell (see (22) ) that (30) amounts to ensuring that, for any $1 \leq k \leq p$, we have

$$
\phi\left(E_{k}^{\prime}\right)=\phi\left(E_{n-p+k-\lambda_{k}} \cap L\right) \subset\left(L+F_{n-p+k-\lambda_{k}}\right) / L=F_{d}^{\prime \prime},
$$


where $d=\operatorname{dim}\left(\left(L+F_{n-p+k-\lambda_{k}}\right) / L\right)$. It remains to calculate $d$. Using the fact that $L \in \Omega_{\mu}^{\circ}\left(F_{\bullet}\right)$, together with (23), we obtain:

$$
\begin{aligned}
d & =\operatorname{dim}\left(F_{n-p+k-\lambda_{k}}\right)-\operatorname{dim}\left(F_{n-p+k-\lambda_{k}} \cap L\right) \\
& =n-p+k-\lambda_{k}-\#\left\{j \in J \mid j>\lambda_{k}+p-k\right\} \\
& =n-p+k-\lambda_{k}-\#\left\{\ell \mid \mu_{\ell}+p+1-\ell>\lambda_{k}+p-k\right\} \\
& =n-p-\lambda_{k}^{*},
\end{aligned}
$$

as claimed. (In fact, this calculation is exactly what determines/defines $\lambda^{*}$ and $\mu^{*}$, and is the only place where we need to know what they are.)

Acknowledgments. We thank Anders Buch for providing experimental evidence for Conjecture 5.1. and therefore for Theorem 1.2 before the latter was proved. We also thank Prakash Belkale for sending us an early version of [1], which motivated the reasoning in Section 5. Finally, we are grateful to the referee for suggesting a number of improvements, including a simplification of the proof of Proposition 2.9.

\section{REFERENCES}

[1] P. Belkale, Geometric proofs of Horn and saturation conjectures, preprint math . AG/0208107.

[2] A. Buch, Littlewood-Richardson calculator, available at http://home.imf .au.dk/abuch/.

[3] C. Carré and B. Leclerc, Splitting the square of a Schur function into its symmetric and antisymmetric parts, J. Algebraic Combin., 4 (1995), 201-231.

[4] S. Fomin, Knuth equivalence, jeu de taquin, and the Littlewood-Richardson rule, Appendix 1 to Chapter 7 in [16], 413-439.

[5] S. Fomin and D. Stanton, Rim hook lattices, St. Petersburg Math. J. 9 (1998), 1007-1016.

[6] W. Fulton, Young Tableaux, Cambridge University Press, 1997.

[7] W. Fulton, Eigenvalues, invariant factors, highest weights, and Schubert calculus, Bull. Amer. Math. Soc., 37 (2000), 209-249.

[8] W. Fulton, Eigenvalues of majorized Hermitian matrices, and Littlewood-Richardson coefficients, Linear Algebra Appl., 319 (2000), 23-36.

[9] A. Horn, Eigenvalues of sums of Hermitian matrices, Pacific J. Math., 12 (1962), 225-241.

[10] A. A. Klyachko, Stable bundles, representation theory and Hermitian operators, Selecta Math. (N.S.) 4 (1998), 419-445.

[11] A. A. Klyachko, Random walks on symmetric spaces and inequalities for matrix spectra, Linear Algebra Appl. 319 (2000), 37-59.

[12] M. A. A. van Leeuwen, Some bijective correspondences involving domino tableaux, Electron. J. Combin. 7 (2000), no. 1, Research Paper 35, 25 pp. (electronic).

[13] C.K. Li and Y.T. Poon, Off-diagonal submatrices of a Hermitian matrix, Proc. Amer. Math. Soc., to appear.

[14] T. Nakayama, On some modular properties of irreducible representations of symmetric groups. I, II. Jap. J. Math. 17 (1940), 165-184, 411-423.

[15] L. O'Shea and R. Sjamaar, Moment maps and Riemannian symmetric pairs, Math. Ann., 317 (2000), 415-457.

[16] R. P. Stanley, Enumerative Combinatorics, vol. 2, Cambridge University Press, 1999.

[17] R. C. Thompson, Principal submatrices IX: Interlacing inequalities for singular values of submatrices, Linear Algebra Appl., 5 (1972), 1-12. 
[18] R. C. Thompson, Singular values, diagonal elements, and convexity, SIAM J. Appl. Math. 32 (1977), no. 1, 39-63.

Department of Mathematics, University of Michigan, Ann Arbor, Mi 04109

E-mail address: fomin@umich.edu

Department of Mathematics, University of Michigan, Ann Arbor, Mi 04109

E-mail address: wfulton@umich.edu

Department of Mathematics, College of William and Mary, Williamsburg, VA 23187

E-mail address: ckli@math.wm.edu

Department of Mathematics, Iowa State University, Ames, IA 50011

Current address: Department of Mathematics, Hong Kong Institute of Education, 10 Lo Ping Road, Tai Po, N. T., Hong Kong

E-mail address: ytpoon@iastate.edu, ytpoon@ied.edu.hk 